Chinese Journal of Organic Chemistry

\title{
芳腈化合物的成对电化学合成
}

\author{
曹志成刘建超褚有群赵峰鸣朱英红* 余远斌*
}

(浙江工业大学化学工程学院 杭州 310032)

\begin{abstract}
摘要 采用循环伏安法 $(\mathrm{CV})$ 和恒电流电解法 $(\mathrm{CCE})$ 研究了对甲氧基苯甲醇 $\left(p-\mathrm{MeOC}_{6} \mathrm{H}_{4} \mathrm{CH}_{2} \mathrm{OH}\right)$ 电化学氧化氰化合成相 应芳腈化合物. 考察了水的体积分数、硫酸浓度、电解温度、电极材料、电流密度和溶剂等因素对该电化学氧化氰化 行为的影响. 结果显示, 以四丁基高氯酸铵 $\left(\mathrm{Bu}_{4} \mathrm{NClO}_{4}\right)$ 为电解质, 差胺 $(\mathrm{HAM})$ 为氮源, 在含 $0.15 \mathrm{~mol} \cdot \mathrm{L}^{-1} \mathrm{H}_{2} \mathrm{SO}_{4}$ 及 $30 \%$ 水的 DMSO 体系中, 电流密度为 $10 \mathrm{~mA} / \mathrm{cm}^{2}$ 条件下恒温 $60{ }^{\circ} \mathrm{C}$ 电解 $8 \mathrm{~h}$, 对甲氧基苯腈 $(p-\mathrm{MeOBN})$ 的收率为 $90 \%$. 通过 对反应底物普适性研究，发现含不同对位和邻位取代基的芳香伯醇均可通过电氧化氰化反应有效转化为相应的腈 (61\% 92\%), 并提出了该电氧化氰化反应的可能机理. 本工作提出了一种在一室型无隔膜电解槽中, 以原位生成醛的 方式, 无需加入额外的催化剂, 通过阴阳两极协同反应的成对电化学合成腈类化合物的方法.

关键词 芳香伯醇; 芳腈化合物; 直接电氧化; 成对电合成
\end{abstract}

\section{Paired Electro-synthesis of Aryl Nitriles}

\author{
Cao, Zhicheng Liu, Jianchao Chu, Youqun Zhao, Fengming \\ Zhu, Yinghong* She, Yuanbin* \\ (College of Chemical Engineering, Zhejiang University of Technology, Hangzhou 310032)
}

\begin{abstract}
The electro-oxidation cyanation of $p$-methoxybenzyl alcohol ( $p$ - $\left.\mathrm{MeOC}_{6} \mathrm{H}_{4} \mathrm{CH}_{2} \mathrm{OH}\right)$ to prepare aryl nitriles was studied by using cyclic voltammetry $(\mathrm{CV})$ and constant current electrolysis $(\mathrm{CCE})$. The effects of the volume ratio of $\mathrm{H}_{2} \mathrm{O}$, concentration of $\mathrm{H}_{2} \mathrm{SO}_{4}$, temperature, electrode materials, current density and solvents on the electro-chemical reaction were studied. The results showed that the yield of $p$-methoxylbenzonitrile ( $p$-MeOBN) was $90 \%$ in $0.15 \mathrm{~mol} \cdot \mathrm{L}^{-1} \mathrm{H}_{2} \mathrm{SO}_{4}$ and $30 \%$ $\mathrm{H}_{2} \mathrm{O}$-DMSO solution at $60{ }^{\circ} \mathrm{C}$ and $10 \mathrm{~mA} / \mathrm{cm}^{2}$ of current density when tetrabutylammonium perchlorate $\left(\mathrm{Bu}_{4} \mathrm{NClO}_{4}\right)$ was used as electrolyte. The CCE of aromatic benzyl alcohols with different $p$ - and $o$-substituted analogs was investigated under the optimized reaction conditions, and the yields toward formation of the corresponding aryl nitriles were $61 \% \sim 92 \%$. A plausible mechanism for the electro-oxidation cyanation procedure was proposed. A novel paired electrochemical method for the synthesis of aryl nitriles from aromatic benzyl alcohols with hydroxylamine (HAM) as "N" source and aldehyde in situ in undivided electrochemical cell was successfully developed.

Keywords aromatic benzyl alcohols; aryl nitriles; direct electro-oxidation; paired electro-synthesis
\end{abstract}

芳腈化合物作为一种重要的化工原料和有机合成 中间体，在农药、医药、染料和功能材料等领域具有广 泛的应用价值 ${ }^{[1 \sim 5]}$, 其合成方法一直受到相关研究者的 广泛关注. 传统的氰化方法包括经典的 Sandmeyer 反 应 $^{[6]}$ 和 Rosenmund-vonBraun 反应 ${ }^{[7]}$, 以及过渡金属催化 卤代芳烃与氰基化试剂的偶联反应 ${ }^{[8 \sim 12]}$, 这些反应大都 涉及毒性较高的氰化试剂, 反应条件苛刻、不易操作. 通过酰氨/醛肟的脱水反应 ${ }^{[13,14]}$ 是合成腈类化合物常用
的方法, 此类反应大都需要在强酸或强碱的条件下进 行, 并需使用大量的脱水试剂. 芳烃的气相氨氧化法是 工业上合成芳腈化合物应用最广泛的方法之一 ${ }^{[16 ~ 18]}$, 该方法不需要特殊溶剂参与, 但是反应过程中高温 $(>$ $\left.350{ }^{\circ} \mathrm{C}\right)$ 、高压及气体的强腐蚀性仍是需待解决的问题. 过渡金属催化 $\mathrm{C}-\mathrm{H}$ 键活化氧基化反应合成腈类化合物 受到了广泛研究 ${ }^{[19-26]}$, 反应底物无需预活化，具有原子 经济性等优点, 但贵金属催化剂的循环利用、开发与氧

\footnotetext{
* Corresponding authors. E-mail: yhzhuchem@zjut.edu.cn; sheyb@zjut.edu.cn Received March 24, 2019; revised April 30, 2019; published online May 15, 2019.

Project supported by the National Key Research and Development Program of China (No. 2017YFB0307503) and the National Program on Key Basic Research Project (973 Program, No. 2012CB722604).

国家重点研发计划(No. 2017YFB0307503)和国家重点基础研究发展规划(973 计划, No. 2012CB722604)资助项目.
} 
化剂相容的氮源等仍是需面临的问题.

目前以醇为原料，通过 “氧化氮化” 法直接合成腈 类化合物已有很多报道 ${ }^{[27]}$, 该法主要包含三个过程: (1) 醇在氧化剂作用下氧化生成相应的醛; (2)醛与氮源发生 氮化反应; (3)重排生成相应的腈. 氧化剂主要有 $\mathrm{I}_{2} /$ $\mathrm{DHI}^{[28,29]} 、 \mathrm{DDQ}^{[30]} 、 \mathrm{TEMPO} / \mathrm{PhI}(\mathrm{OAc})_{2}{ }^{[31,32]}$ 以及金属氧 化物 $\mathrm{MnO}_{2} / \mathrm{Al}_{2} \mathrm{O}_{3}{ }^{[33,34]}$ 等, 氮源有氨气 ${ }^{[35]}$ 、铵盐 ${ }^{[36]}$ 等 (Scheme 1 (1)). 因此, 氧化剂与氮源的适应性成为反应 条件优化的关键.

有机电合成已成为有机合成中的一个重要组成部 分, 通过 $\mathrm{C}-\mathrm{H}$ 键电化学活化构建 $\mathrm{C}-\mathrm{X}(\mathrm{X}=\mathrm{C} 、 \mathrm{~N} 、 \mathrm{O}$ 、 S)键合成具有高附加值的化学品受到了广泛研究 ${ }^{[37]}$, 因此利用电子作为氧化还原试剂合成腈类化合物极具 吸引力. Yuan 等 ${ }^{[38]}$ 报道了以 $\mathrm{NH}_{4} \mathrm{I}$ 作为电催化剂和氮源, 实现了从醛到腈的间接电化学合成, 该方法简单高效, 但碘化铵用量较大对环境不友好. $\mathrm{Li}$ 等 ${ }^{[39]}$ 报道了以 2,2 , 6,6-四甲基哌啶氮氧基自由基(TEMPO)作为电催化剂, 醋酸铵 $\left(\mathrm{NH}_{4} \mathrm{OAc}\right)$ 作为氮源, 实现了从芳醇到腈的间接 电氧化合成(Scheme 1(2)). 目前, 通过醇直接电氧化氰 化反应合成腈类化合物未见文献报道.

在醇/醛电化学氰化工作的启发下, 本文提出了由 芳醇直接电化学氧化生成醛, 再进一步氰化转化为腈的 电化学合成法(Scheme 1(3)). 以羟胺为反应氮源, 四丁 基高氯酸铵 $\left(\mathrm{Bu}_{4} \mathrm{NClO}_{4}\right)$ 为支持电解质, 采用恒电流电解 技术, 研究了从芳香伯醇到芳腈的直接电氧化氰化合成 过程, 这种在阴阳两极同时发生电化学转化的成对电氧 化还原合成路线极大简化了反应步骤.

\section{Previous reports}<smiles>OCC1=CC=[R]=CC=C1</smiles>
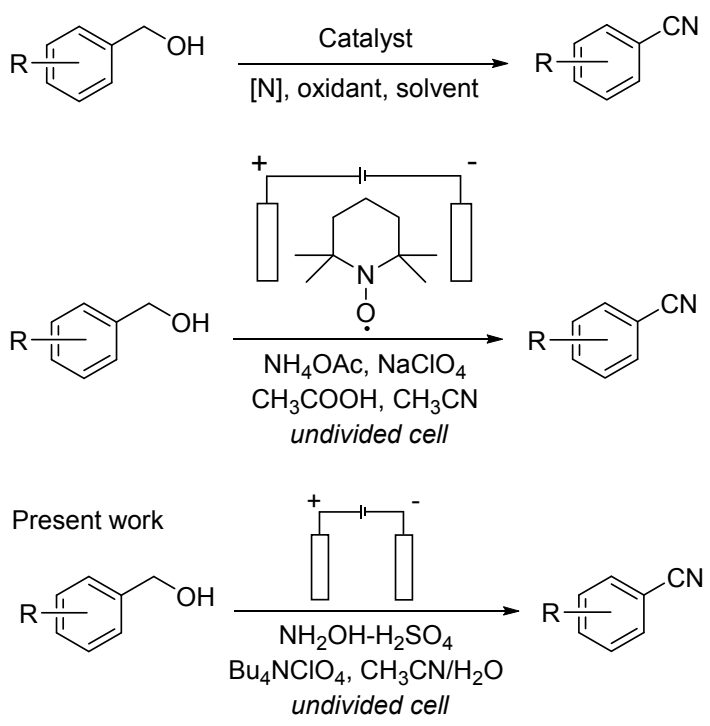

图式 1 芳香伯醇到芳腈的合成路线

Scheme 1 Synthesis of aryl nitriles from aromatic benzyl alcohols

\section{1 结果与讨论}

\section{$1.1 p-\mathrm{MeC}_{6} \mathrm{H}_{4} \mathrm{CH}_{2} \mathrm{OH}$ 的循环伏安 $(\mathrm{CV})$ 测试}

在以 $0.05 \mathrm{~mol} \cdot \mathrm{L}^{-1} \mathrm{Bu}_{4} \mathrm{NClO}_{4}$ 作为支持电解质的情 况下，考察了水的体积含量对 $p-\mathrm{MeOC}_{6} \mathrm{H}_{4} \mathrm{CH}_{2} \mathrm{OH}$ 电氧 化行为的影响, 结果如图 1 所示. 在没有额外加入水的 情况下, $1.60 \mathrm{~V}$ 处出现微弱的氧化峰. 当水体积含量为 $10 \%$ 时, 氧化峰电流明显增加, 氧化峰电位正移到 1.74 $\mathrm{V}$ 左右. 而随着水含量继续增加, $p-\mathrm{MeOC}_{6} \mathrm{H}_{4} \mathrm{CH}_{2} \mathrm{OH}$ 的 氧化电位发生负移. 当水含量增加到 $30 \%$ 时, $p-\mathrm{MeO}$ $\mathrm{C}_{6} \mathrm{H}_{4} \mathrm{CH}_{2} \mathrm{OH}$ 的氧化峰电位负移到 $1.63 \mathrm{~V}$ 左右, 由于这 种比例的水与乙腈的混合溶剂与反应底物之间达到了 一种互溶状态，有利于反应底物的扩散，加快了电极与 活性物种之间电荷传递速率, 峰电流增强, 峰电位下降. 当水含量超过 $40 \%$ 后, $p$ - $\mathrm{MeOC}_{6} \mathrm{H}_{4} \mathrm{CH}_{2} \mathrm{OH}$ 的氧化峰电流 有所下降，因为过多的水导致有机反应底物在混合溶剂 中溶解度下降，从而使电氧化性能下降.

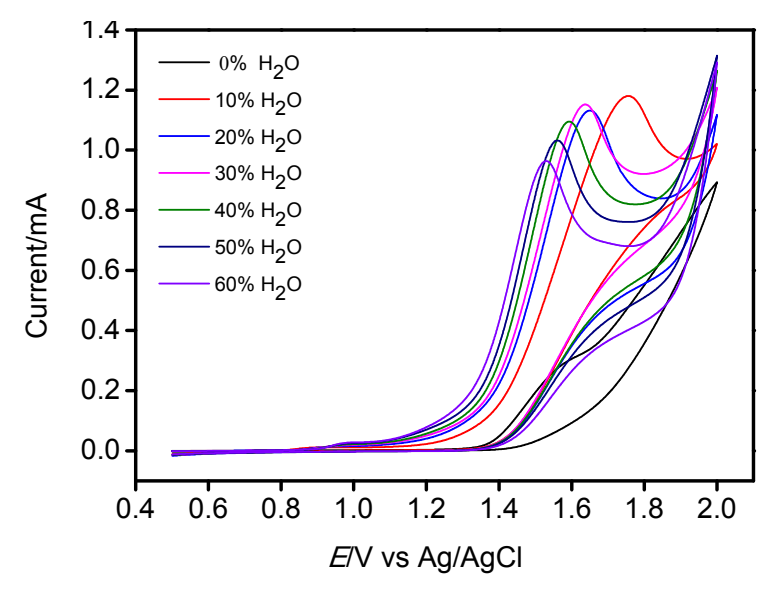

图 1 不同水体积含量下 $p-\mathrm{MeOC}_{6} \mathrm{H}_{4} \mathrm{CH}_{2} \mathrm{OH}\left(0.05 \mathrm{~mol} \cdot \mathrm{L}^{-1}\right)$ 电 氧化循环伏安曲线 $\left(v=50 \mathrm{mV} \cdot \mathrm{s}^{-1}\right)$

Figure 1 Cyclic voltammograms of $p$ - $\mathrm{MeOC}_{6} \mathrm{H}_{4} \mathrm{CH}_{2} \mathrm{OH}$ at different volume proportion of $\mathrm{H}_{2} \mathrm{O}\left(v=50 \mathrm{mV} \cdot \mathrm{s}^{-1}\right)$

图 2 是不同硫酸浓度对 $p-\mathrm{MeOC}_{6} \mathrm{H}_{4} \mathrm{CH}_{2} \mathrm{OH}$ 电氧化 行为的影响. 发现体系中的酸性强弱对 $p-\mathrm{MeOC}_{6} \mathrm{H}_{4}-$ $\mathrm{CH}_{2} \mathrm{OH}$ 电氧化过程的峰电流和峰电位均有较大的影响. 无硫酸加入时, 在 $1.83 \mathrm{~V}$ 左右出现氧化峰. 在硫酸浓度 $\leqslant 0.35 \mathrm{~mol} \cdot \mathrm{L}^{-1}$ 时，随着硫酸浓度的增加， $p-\mathrm{MeO}-$ $\mathrm{C}_{6} \mathrm{H}_{4} \mathrm{CH}_{2} \mathrm{OH}$ 的氧化电位发生负移, 氧化峰电流逐步增 强, 这是由于溶液的离子强度增强所致, 同时体系中 $\mathrm{H}^{+}$浓度增大，有利于阴极上析氢反应的进行。当硫酸浓 度为 $0.35 \mathrm{~mol} \cdot \mathrm{L}^{-1}$ 时, $p-\mathrm{MeOC}_{6} \mathrm{H}_{4} \mathrm{CH}_{2} \mathrm{OH}$ 的氧化电位为 $1.57 \mathrm{~V}$ 左右. 随着硫酸浓度进一步增加, $p-\mathrm{MeOC}_{6} \mathrm{H}_{4}-$ $\mathrm{CH}_{2} \mathrm{OH}$ 的氧化峰电流出现微弱下降, 表明适量的 $\mathrm{H}^{+}$浓 度有利于 $p-\mathrm{MeOC}_{6} \mathrm{H}_{4} \mathrm{CH}_{2} \mathrm{OH}$ 阳极氧化反应的进行.

在 $0.15 \mathrm{~mol} \cdot \mathrm{L}^{-1} \mathrm{H}_{2} \mathrm{SO}_{4}$ 和 $30 \% \mathrm{H}_{2} \mathrm{O}$ 的情况下考察不 
同温度对 $p-\mathrm{MeOC}_{6} \mathrm{H}_{4} \mathrm{CH}_{2} \mathrm{OH}$ 的电氧化行为的影响, 见 图 3. 研究发现, 随着温度的升高, 氧化峰电流逐渐增 大, 氧化峰电位有所负移. 说明提高温度在一定程度上 能加快反应底物向电极表面的扩散, 有利于 $p$ - MeO$\mathrm{C}_{6} \mathrm{H}_{4} \mathrm{CH}_{2} \mathrm{OH}$ 的电氧化过程. 考虑到羟胺在高温下容易 分解以及腈类化合物的水解反应, 没有继续研究更高温 度下的影响, 因此在后续恒电流电解反应中最高电解温 度控制在 $60{ }^{\circ} \mathrm{C}$.

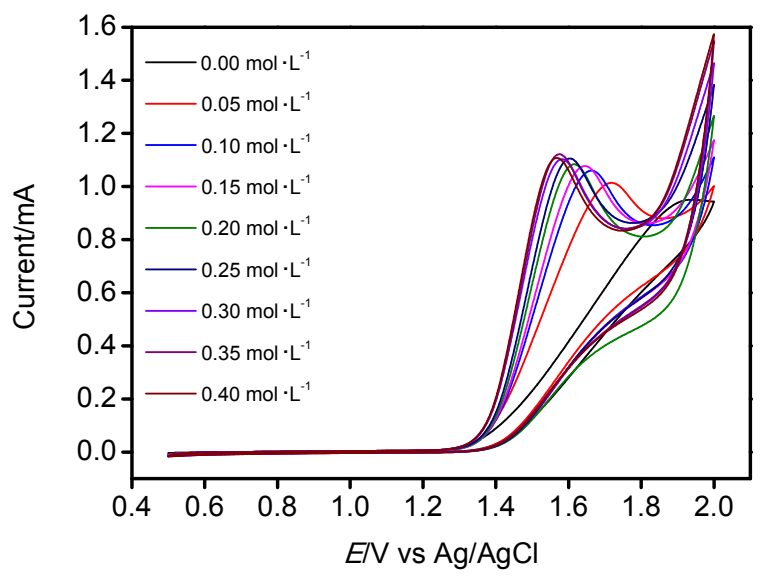

图 2 不同硫酸浓度下 $p-\mathrm{MeOC}_{6} \mathrm{H}_{4} \mathrm{CH}_{2} \mathrm{OH}\left(0.05 \mathrm{~mol} \cdot \mathrm{L}^{-1}\right)$ 电氧 化循环伏安曲线 $\left(v=50 \mathrm{mV} \cdot \mathrm{s}^{-1}\right)$

Figure 2 Cyclic voltammograms of $p-\mathrm{MeOC}_{6} \mathrm{H}_{4} \mathrm{CH}_{2} \mathrm{OH}$ at different concentration of $\mathrm{H}_{2} \mathrm{SO}_{4}\left(v=50 \mathrm{mV} \cdot \mathrm{s}^{-1}\right)$

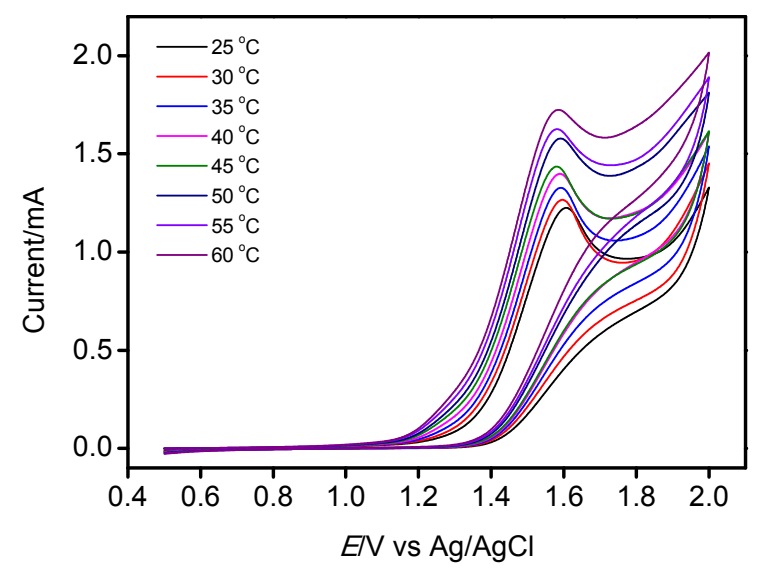

图 3 不同温度下 $p-\mathrm{MeOC}_{6} \mathrm{H}_{4} \mathrm{CH}_{2} \mathrm{OH}\left(0.05 \mathrm{~mol} \cdot \mathrm{L}^{-1}\right)$ 电氧化 循环伏安曲线 $\left(v=50 \mathrm{mV} \cdot \mathrm{s}^{-1}\right)$

Figure 3 Cyclic voltammograms of $p-\mathrm{MeOC}_{6} \mathrm{H}_{4} \mathrm{CH}_{2} \mathrm{OH}$ at different temperature $\left(v=50 \mathrm{mV} \cdot \mathrm{s}^{-1}\right)$

\section{2 恒电流电解实验条件的优化}

为了进一步探究 $p-\mathrm{MeOC}_{6} \mathrm{H}_{4} \mathrm{CH}_{2} \mathrm{OH}$ 电氧化氰化反 应的最佳反应条件, 在 $\mathrm{CV}$ 的研究基础上, 进行了恒电 流电解实验, 考察了支持电解质浓度、电流密度、电极 材料、反应温度、酸浓度、溶剂等对 $p-\mathrm{MeOC}_{6} \mathrm{H}_{4} \mathrm{CH}_{2} \mathrm{OH}$ 转化率和目标产物对甲氧基苯腈 $(p-\mathrm{MeOBN})$ 收率的影
响, 结果列于表 1 中.

在有机电合成过程中, 需要加入支持电解质提高体 系的导电性确保反应的顺利进行. 在不同 $\mathrm{Bu}_{4} \mathrm{NClO}_{4}$ 浓 度下, $p-\mathrm{MeOC}_{6} \mathrm{H}_{4} \mathrm{CH}_{2} \mathrm{OH}$ 电氧化氰化反应的结果见表 1 Entries 1 4. 结果表明, 在加入不同当量的支持电解质 下, $p-\mathrm{MeOC}_{6} \mathrm{H}_{4} \mathrm{CH}_{2} \mathrm{OH}$ 均具有良好的转化率 $(85 \%$ $99 \%$ ), 但对 $p$ - MeOBN 的选择性影响较大. 在加入 0.3 equiv. 的 $\mathrm{Bu}_{4} \mathrm{NClO}_{4}$ 时, $p-\mathrm{MeOBN}$ 的收率仅为 $40 \%$. 随着 支持电解质当量的增加，收率从 $40 \%$ 提高到 $83 \%$ ，但考 虑到过量的 $\mathrm{Bu}_{4} \mathrm{NClO}_{4}$ 使得体系的黏度增加, 阻碍反应 物种的扩散，同时目标产物的收率也有所下降，因此选 择 1.0 equiv.的 $\mathrm{Bu}_{4} \mathrm{NClO}_{4}$ 为最佳用量.

随着电流密度的增大, $p-\mathrm{MeOC}_{6} \mathrm{H}_{4} \mathrm{CH}_{2} \mathrm{OH}$ 可以有效 转化 (转化率 $\geqslant 96 \%$ ), 但 $p$-MeOBN 的收率却有所下降 (表 1, Entries 3, 5 6). 通过 GC-MS 检测, 发现随着电流 密度的增加, 产物中醛的进一步氧化产物对甲氧基苯甲 酸的量也增加 ${ }^{[40,41]}$.

温度是影响反应底物在体系中溶解度及扩散速率 的重要因素, 其对 $p-\mathrm{MeOC}_{6} \mathrm{H}_{4} \mathrm{CH}_{2} \mathrm{OH}$ 电氧化氰化反应 的影响见表 1 (Entries 3, 7 9). 结果发现, 随着温度的 升高, $p-\mathrm{MeOC}_{6} \mathrm{H}_{4} \mathrm{CH}_{2} \mathrm{OH}$ 的转化率和 $p-\mathrm{MeOBN}$ 的收率 均明显提高.

电极材料是电化学反应的场所, 不同阴极材料对 $p-\mathrm{MeOC}_{6} \mathrm{H}_{4} \mathrm{CH}_{2} \mathrm{OH}$ 电氧化氧化行为的影响见表 1 (Entries $3,10,11)$. 以 $\mathrm{Pt}$ 作为阳极, 在三种阴极材料上, $p$ $\mathrm{MeOC}_{6} \mathrm{H}_{4} \mathrm{CH}_{2} \mathrm{OH}$ 的转化率很高( $\geqslant 97 \%$ ), 但 $p$-MeOBN 的收率却有较大差别. 当石墨作为阴极时, $p-\mathrm{MeOBN}$ 的 收率仅为 $42 \%$. 以铂作为阴极时, 收率提高到 $78 \%$. 以 铅作为阴极时, 收率明显提高到 $83 \%$. 显然, 与铂或石 墨相比, 铅是一种适合电氧化氰化反应的阴极材料, 同 时也表明 $p-\mathrm{MeOC}_{6} \mathrm{H}_{4} \mathrm{CH}_{2} \mathrm{OH}$ 电氧化氰化过程中, 在阴 极上除了析氢反应, 还涉及一步反应中间产物的转化过 程, 这将在后续的机理探讨中进行佐证.

溶剂既影响反应底物在体系中的溶解性能, 也影响 电化学反应的后续步骤, 因此电合成反应的选择性受溶 剂的影响较大. 对比 $p-\mathrm{MeOC}_{6} \mathrm{H}_{4} \mathrm{CH}_{2} \mathrm{OH}$ 在不同溶剂中 电氧化氰化反应结果(表 1 , Entries $3,12 \sim 15$ ), 发现在不 同溶剂中, $p-\mathrm{MeOC}_{6} \mathrm{H}_{4} \mathrm{CH}_{2} \mathrm{OH}$ 的转化率都很高 $(\geqslant 90 \%)$, 但 $p$ - $\mathrm{MeOBN}$ 的收率差别较大. 在甲醇溶剂中副反应明 显，在 GC-MS 检测中发现有 56\%的甲氧基化产物. 在 乙醇和乙腈溶剂中, $p$-MeOBN 的收率明显提高, 但在产 物中检测到一定量的相应酸和朊. 当 DMSO 作溶剂时, $p$ - $\mathrm{MeOBN}$ 的收率最高为 $90 \%$.

在酸浓度对 $p$ - $\mathrm{MeOC}_{6} \mathrm{H}_{4} \mathrm{CH}_{2} \mathrm{OH}$ 电氧化影响的 $\mathrm{CV}$ 曲线中(图2), 发现随着酸浓度的增加, 氧化峰电流逐渐 
表 $1 p-\mathrm{MeOC}_{6} \mathrm{H}_{4} \mathrm{CH}_{2} \mathrm{OH}$ 电合成 $p-\mathrm{MeOBN}$ 反应条件的优化 ${ }^{a}$

Table 1 Optimization of the electro-synthesis of $p$-MeOBN from $p$ - $\mathrm{MeOC}_{6} \mathrm{H}_{4} \mathrm{CH}_{2} \mathrm{OH}$

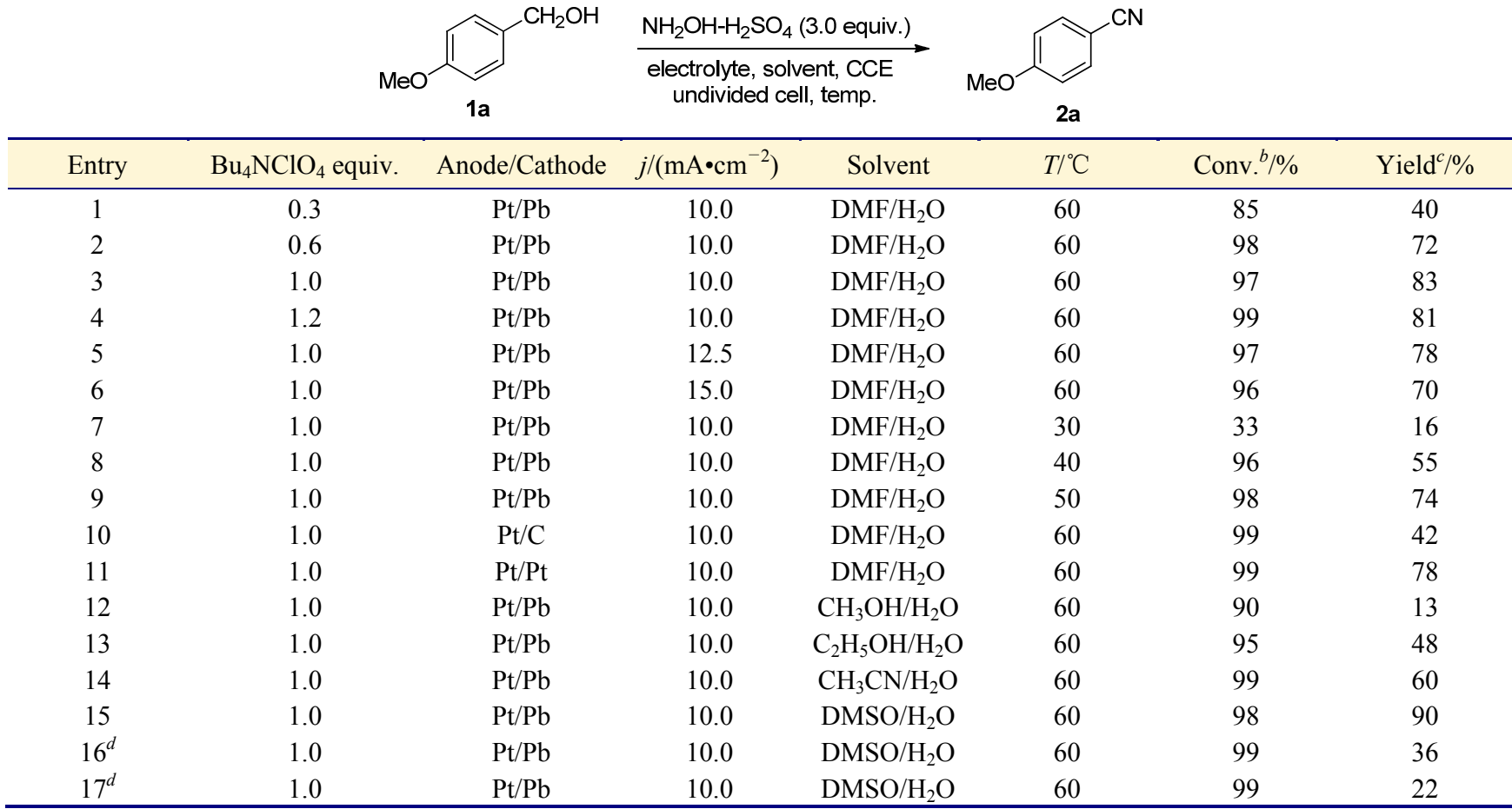

${ }^{a} p-\mathrm{MeOC}_{6} \mathrm{H}_{4} \mathrm{CH}_{2} \mathrm{OH}(1.5 \mathrm{mmol}), \mathrm{NH}_{2} \mathrm{OH}\left(3.0\right.$ equiv), $\mathrm{H}_{2} \mathrm{SO}_{4}\left(0.15 \mathrm{~mol} \cdot \mathrm{L}^{-1}\right)$, solvent $/ \mathrm{H}_{2} \mathrm{O}(30 \%, 30 \mathrm{~mL})$ was electrolyzed at a constant current in an undivided cell $t=8 \mathrm{~h}, \mathrm{Pt} / \mathrm{Pb}(2 \mathrm{~cm} \times 2 \mathrm{~cm}) ;{ }^{b}$ Conversions were determined by GC; ${ }^{c} \mathrm{GC}$ yields of nitriles; ${ }^{d} \mathrm{H}_{2} \mathrm{SO}_{4}\left(0.25,0.35 \mathrm{~mol} \cdot \mathrm{L}^{-1}\right)$.

增大. 但通过恒电流电解发现, 当酸浓度大于 0.15 $\mathrm{mol} \cdot \mathrm{L}^{-1}$ 时, $p-\mathrm{MeOC}_{6} \mathrm{H}_{4} \mathrm{CH}_{2} \mathrm{OH}$ 的转化率高达 $99 \%$, 而 $p$-MeOBN 的收率却明显下降(表 1, Entries 3,16,17), 在 产物中检测到约 $50 \%$ 的醛及相应酸, 这说明在强酸性条 件下不仅使醛容易进一步氧化, 也使阴极的析氢反应加 剧, 从而影响醛与羟胺缩合后的中间体进一步转化为相 应腈的步骤, 致使反应停留在醛的这一步. 这也进一步 说明了阴极电极材料对 $p-\mathrm{MeOC}_{6} \mathrm{H}_{4} \mathrm{CH}_{2} \mathrm{OH}$ 电氧化氰化 反应产生影响的原因. 这些结果表明, $p-\mathrm{MeOC}_{6} \mathrm{H}_{4} \mathrm{CH}_{2}$ $\mathrm{OH}$ 电氧化氰化合成相应腈的反应很可能是一个同时利 用阳极和阴极的成对电化学合成反应.

\section{3 反应底物的普适性研究}

基于以上结果, 对不同取代基取代的芳香伯醇进行 了普适性研究, 在实验范围内, 将不同反应底物分别在 溶剂 DMSO 和 $\mathrm{CH}_{3} \mathrm{CN}$ 中取得的较好电解结果列于表 2 中. 不论是供电子基团 $(\mathrm{OMe}, \mathrm{Me}, t-\mathrm{Bu})$ 还是弱吸电子基 团 $(\mathrm{F}, \mathrm{Cl}, \mathrm{Br})$, 取代位置为邻、对位时, 芳香伯醇在电氧 化氰化反应中均具有很好的转化率 $(\geqslant 96 \%)$, 其相应目 标产物腈的收率在 79\% 92\%. 当取代基为强吸电子基 团 $\mathrm{CF}_{3}$ 时, 转化率有所下降 $(93 \%)$, 相应目标产物对三氟 甲基苯甲腈的收率为 $61 \%$ (表 2, Entry 12). 而间位取代 的芳香醇在转化率和产物收率上比邻、对位取代的效果 差(表 2, Entries 3,9), 这可能是由于 p-П共轭效应的影 响, 使得邻、对位取代的芳香醇在直接电氧化过程中生
成的鎓阳正离子更稳定，有利于反应的顺利进行.

直接以不同对位取代的芳香醛为反应底物进行电 氧化氰化反应, 结果列于表 3 中. 供电子基 $(\mathrm{OMe}, \mathrm{Me})$ 和吸电子基 $(\mathrm{F})$ 取代的醛，该电氧化氰化反应的转化率 都很高 $(\geqslant 95 \%)$, 主要产物为相应的芳腈, 但收率比以 相同取代基的醇为反应底物时要低, 其中供电子基取代 的醛在电氧化氧化过程中易进一步生成相应的酸, 而吸 电子基取代的醛在产物中还有其他物质生成. 这可能是 由于在酸性条件下，电极表面高浓度的醛更容易进一步 失去电子氧化为相应的酸, 以及由于醛与羟胺很容易生 成肟, 从而增加了高浓度的肜与乙腈等发生其他副反应 的机会 ${ }^{[42]}$. 因此在本实验中通过原位生成酫，进一步发 生氰基化反应是一种更有效的氰化方法.

\section{4 反应机理探讨}

为了佐证电流对 $p-\mathrm{MeOC}_{6} \mathrm{H}_{4} \mathrm{CH}_{2} \mathrm{OH}$ 电氧化性能的 影响, 在一室型电解槽中不通电流情况下, 发现 $p-\mathrm{MeOC}_{6} \mathrm{H}_{4} \mathrm{CH}_{2} \mathrm{OH}$ 几乎未发生转化, 说明电流是芳香 醇发生电氧化反应的必要条件. 根据不同的阴极材料及 体系中酸性强弱对电解产物的影响结果, 推测在阴极除 了析氢反应，还有中间体的还原反应，进而生成目标产 物 $p$-MeOBN. 为了佐证这一假设, 在其他条件一样的 情况下, 采用 $\mathrm{H}$ 型隔膜电解槽分别在阴极室和阳极室进 行 $p-\mathrm{MeOC}_{6} \mathrm{H}_{4} \mathrm{CH}_{2} \mathrm{OH}$ 的恒电流电解实验, 发现在阴极 室反应底物几乎未发生转化. 而在阳极室中，主要检测 
表 2 不同取代芳香伯醇电氧化氰化合成腈的反应 ${ }^{a}$

Table 2 Electro-oxidation cyanation of aromatic primary alcohols to corresponding nitriles

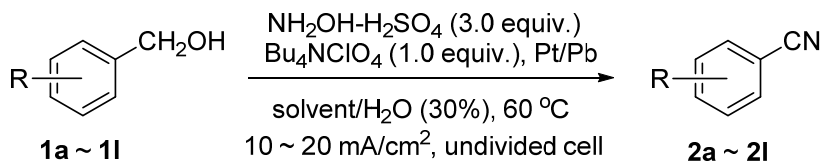

\begin{tabular}{|c|c|c|c|c|c|}
\hline Entry & Product & $j /\left(\mathrm{mA} \cdot \mathrm{cm}^{-2}\right)$ & Time/h & Conv. ${ }^{b} / \%$ & Yield $^{c} / \%$ \\
\hline 1 & $2 a$ & 10.0 & 10 & 99 & 90 \\
\hline 2 & $2 b$ & 17.5 & 9 & 99 & 85 \\
\hline 3 & $2 c$ & 17.5 & 13 & 59 & 15 \\
\hline 4 & $2 d$ & 17.5 & 13 & 99 & 92 \\
\hline 5 & $2 e$ & 12.5 & 9 & 99 & 89 \\
\hline 6 & $2 f$ & 12.5 & 11 & 96 & 85 \\
\hline 7 & $2 g$ & 17.5 & 10 & 97 & 89 \\
\hline 8 & $2 h$ & 20.0 & 11 & 98 & 90 \\
\hline 9 & 21 & 20.0 & 11 & 89 & 59 \\
\hline 10 & 2j & 20.0 & 11 & 99 & 86 \\
\hline 11 & $2 k$ & 20.0 & 10 & 99 & 79 \\
\hline 12 & 2| & 17.5 & 13 & 93 & 61 \\
\hline
\end{tabular}


表 3 不取代芳香醛电氧化氯化合成腈 ${ }^{a}$

Table 3 Electro-oxidation cyanation of aromatic aldehydes to corresponding nitriles
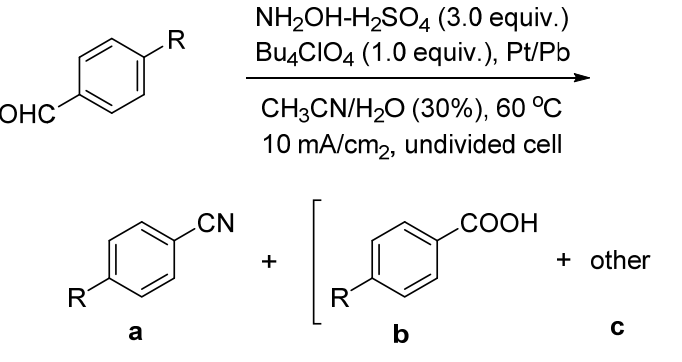

\begin{tabular}{|c|c|c|c|c|c|c|}
\hline \multirow{2}{*}{ Entry } & \multirow{2}{*}{ Substrte } & \multirow{2}{*}{ Time/h } & \multirow{2}{*}{ Conv. ${ }^{b} / \%$} & \multicolumn{3}{|c|}{ Yield $^{c} / \%$} \\
\hline & & & & $\mathbf{a}$ & $\mathbf{b}$ & c \\
\hline 1 & & 8 & 98 & 85 & 9 & n.d. \\
\hline 2 & & 9 & 95 & 78 & 6 & 11 \\
\hline 3 & & 9 & 98 & 75 & n.d. & 23 \\
\hline
\end{tabular}

${ }^{a}$ Aromatic aldehydes (1.5 mmol), $\mathrm{NH}_{2} \mathrm{OH}-\mathrm{H}_{2} \mathrm{SO}_{4}$ (3.0 equiv.), $\mathrm{Bu}_{4} \mathrm{NClO}_{4}(1.0$ equiv.), $\mathrm{Pt}(2 \mathrm{~cm} \times 2 \mathrm{~cm}), \mathrm{Pb}(2 \mathrm{~cm} \times 2 \mathrm{~cm}), j\left(10 \mathrm{~mA} / \mathrm{cm}^{2}\right)$, solvent $/ \mathrm{H}_{2} \mathrm{O}(30 \%$, $30 \mathrm{~mL}$ ), $60{ }^{\circ} \mathrm{C}$, Undivided cell; ${ }^{b}$ Conversions were determined by GC; ${ }^{c} \mathrm{GC}$ yields of nitriles; n.d. $=$ no detection.

到 $86 \%$ 的对甲氧基苯甲醛，这说明 $p-\mathrm{MeOC}_{6} \mathrm{H}_{4} \mathrm{CH}_{2} \mathrm{OH}$ 在阳极发生电化学氧化反应生成 $p$-MeOBA. 当直接以 $p-\mathrm{MeOBA}$ 为反应底物, 采用 $\mathrm{H}$ 型隔膜电解槽在阴极室 进行恒电流电解时, 反应停留在生成对甲氧基苯甲醶肜 $(p-\mathrm{MeOBAO}$, 收率为 $88 \%$ ) 这一步, 而没有进一步转化 为相应的腈.

根据以上实验结果, 我们认为 $p-\mathrm{MeOC}_{6} \mathrm{H}_{4} \mathrm{CH}_{2} \mathrm{OH}$ 在阳极发生氧化反应生成相应醛后, 与羟胺生成 $p$-MeOBAO, 随之在阳极和阴极的共同作用下进而转化 为 $p-\mathrm{MeOBN}$, 可能的反应机理见 Scheme 2 . $p-\mathrm{MeOC}_{6} \mathrm{H}_{4} \mathrm{CH}_{2} \mathrm{OH}$ 在阳极先失去两个电子得到鎓阳离 子 $\mathbf{2}$, 在水溶液中生成氧鎓离子 $\mathbf{3}$, 继而脱去 $\mathrm{H}^{+}$生成缩 醛 4. 由于缩醛极不稳定, 在酸性条件下重排脱掉一分 子水生成活性中间体醛 $\mathbf{5}^{[43,44]}$, 醛与溶液中的羟胺发生 亲核加成反应生成醛肜 6. 然后, 醛肜在阳极作用下进 一步失去两个电子和两个质子生成腈氧化物中间体 $7^{[45]}$, 再经过阴极得到两个电子还原生成目标产物腈 $8^{[46 \sim 48]}$

\section{2 结论}

提出了一种在无隔膜电解槽中以羟胺(HAM)为反 应氮源，芳香醇为反应底物，通过原位生成醛，继而在 阴阳两极上进一步氧化转化的成对电化学合成芳腈化 合物的方法. 以 $\mathrm{Bu}_{4} \mathrm{NClO}_{4}$ 为电解质，在 $0.15 \mathrm{~mol} \cdot \mathrm{L}^{-1}$

$\mathrm{H}_{2} \mathrm{SO}_{4}$ 及 $30 \%$ 水的有机溶剂体系中, 不同取代芳香醇具 有一定的普适性，相应腈的收率在 $61 \sim 92 \%$ 之间。该成 对电氧化还原合成路线极大地缩短了从醇出发合成腈 类化合物的合成步骤.<smiles>COc1ccc(CO)cc1</smiles>

1
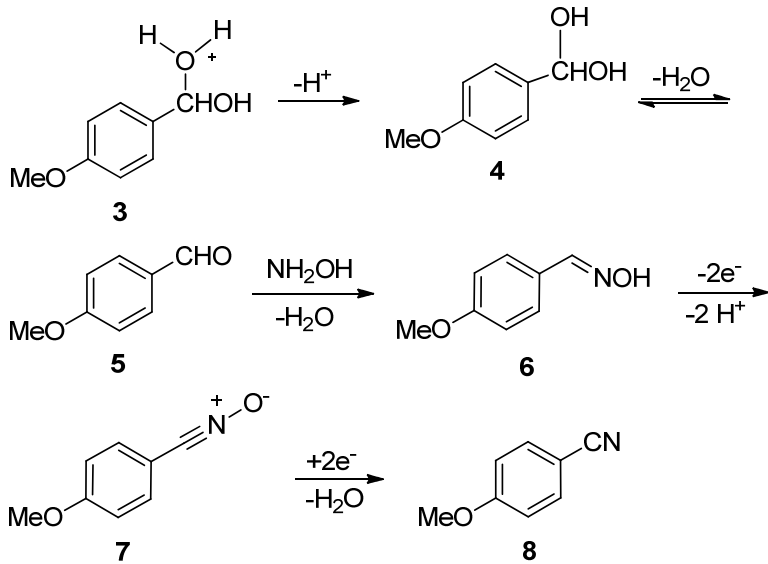<smiles>COc1ccc(C#N)cc1</smiles>

图式 2 可能的电氧化氧化反应机理

Scheme 2 Plausible reaction mechanism of the electro-oxidation cyanidation

\section{3 实验部分}

\section{1 仪器与试剂}

循环伏安测试采用上海辰华电化学工作站 (CHI660D), 恒电流电解采用固纬直流电源 (GWINSTEK GPD-4303S)，产物分析采用气相质谱联用 仪 GC-MS (Thermo Trace ISQ instrument with TG 5MS capillary column), 气相色谱分析仪 GC (Agilent 7890 A with Hp-5 capillary column and a FID), 物质的熔点采用 SGWX-4A 显微熔点仪测定，核磁共振谱由 Bruker-500 $\mathrm{MHz}$ 型核磁共振仪测定, $\mathrm{CDCl}_{3}$ 为溶剂, 分别在 500、125 $\mathrm{MHz}$ 条件下得到 ${ }^{1} \mathrm{H} \mathrm{NMR} 、{ }^{13} \mathrm{C} \mathrm{NMR}$ 谱图. 反应中所用 到的反应原料、溶剂均为市售无水分析纯试剂，电极材 料购自于天津艾达恒晟科技有限公司.

\section{2 实验方法}

循环伏安 $(\mathrm{CV})$ 测试, 在无隔膜的一室型单电解池 $(10 \mathrm{~mL})$ 中采用三电极体系进行. 工作电极 (WE) 为铂电 极 $(\varphi=3 \mathrm{~mm})$, 辅助电极 $(\mathrm{CE})$ 为铂片电极 $(2 \mathrm{~cm} \times 2 \mathrm{~cm})$, 参比电极 $(\mathrm{RE})$ 为 $\mathrm{Ag} / \mathrm{AgCl}$ 电极, 于室温下进行 $\mathrm{CV}$ 测试. 扫描速率为 $50 \mathrm{mV} \cdot \mathrm{s}^{-1}$, 扫描范围为 $0.5 \sim 2.0 \mathrm{~V}$. 溶液为 含有 $0.05 \mathrm{~mol} \cdot \mathrm{L}^{-1}$ 四丁基高氯酸铵的 $\mathrm{DMF}$ 水溶液, 底物 浓度为 $0.05 \mathrm{~mol} \cdot \mathrm{L}^{-1}$

恒电流电解(CCE) 实验在配备磁子搅拌的一室型恒 温电解槽 $(50 \mathrm{~mL})$ 中采用双电极体系进行. 电极材料分 
别为铂片电极 $(2 \mathrm{~cm} \times 2 \mathrm{~cm})$, 铅片电极 $(2 \mathrm{~cm} \times 2 \mathrm{~cm})$, 石 墨棒电极 $(\varphi=3 \mathrm{~mm})$. 电解液为含有体积分数为 $30 \%$ 的 水和 $0.15 \mathrm{~mol} \cdot \mathrm{L}^{-1} \mathrm{H}_{2} \mathrm{SO}_{4}$ 的 DMF 或 DMSO 溶液 $(30 \mathrm{~mL})$, 反应底物为对甲氧基苯甲醇 $\left(0.05 \mathrm{~mol} \cdot \mathrm{L}^{-1}\right)$, 反应氮源为 差弪胺 $\left(0.15 \mathrm{~mol} \cdot \mathrm{L}^{-1}\right)$, 支持电解质为四丁基高氯酸铵 $\left(0.05 \mathrm{~mol} \cdot \mathrm{L}^{-1}\right)$, 反应温度为 $60^{\circ} \mathrm{C}$, 恒电流电解 $8 \mathrm{~h}$. 电解 产物经过无水乙梄萃取 $(20 \mathrm{~mL} \times 3)$, 有机相经过水洗涤 $(20 \mathrm{~mL} \times 3)$, 最后用无水硫酸钠干燥. 产物的定性分析 采用气质联用仪 GC-MS 进行, 定量分析采用气相色谱 分析仪 GC 进行, 分析方法为面积归一化法. 目标产物 经柱层析提纯[展开剂: $V$ (石油醚) $： V($ 乙酸乙酯 $)=15$ : $1]$.

\section{3 化合物结构数据}

所有化合物表征数据均与参考文献一致 ${ }^{[1 \sim 3,12 b, 19,39]}$ 4-甲氧基苯腈 $(2 \mathbf{a})^{[1]}$ : 白色固体, m.p. $58 \sim 59{ }^{\circ} \mathrm{C} ;{ }^{1} \mathrm{H}$ NMR $\left(500 \mathrm{MHz}, \mathrm{CDCl}_{3}\right) \delta: 3.86(\mathrm{~s}, 3 \mathrm{H}), 6.96(\mathrm{~d}, J=8.5$ $\mathrm{Hz}, 2 \mathrm{H}), 7.59$ (d, $J=8.0 \mathrm{~Hz}, 2 \mathrm{H}) ;{ }^{13} \mathrm{C}$ NMR $(125 \mathrm{MHz}$, $\left.\mathrm{CDCl}_{3}\right) \delta: 55.52,104.00,114.76,119.17,133.95,162.86$; GC-MS (EI, $70 \mathrm{eV}$ ) $\mathrm{m} / z: 133.05[\mathrm{M}]^{+}$.

4-甲基苯腈 $(2 \mathbf{b}){ }^{[12 b]}$ : 白色固体, m.p. $34 \sim 35{ }^{\circ} \mathrm{C} ;{ }^{1} \mathrm{H}$ NMR (500 MHz, $\left.\mathrm{CDCl}_{3}\right) \delta: 2.42(\mathrm{~s}, 3 \mathrm{H}), 7.27$ (d, $J=8.0$ $\mathrm{Hz}, 2 \mathrm{H}), 7.53$ (d, $J=8.5 \mathrm{~Hz}, 2 \mathrm{H}) ;{ }^{13} \mathrm{C}$ NMR $(125 \mathrm{MHz}$, $\left.\mathrm{CDCl}_{3}\right) \delta: 21.79,109.37,119.10,129.02$ 132.03, 143.66; GC-MS (EI, $70 \mathrm{eV}$ ) $m / z: 117.16[\mathrm{M}]^{+}$.

3-甲基苯腈(2c) ${ }^{[19]}$ : 无色液体. ${ }^{1} \mathrm{H}$ NMR $(500 \mathrm{MHz}$, $\left.\mathrm{CDCl}_{3}\right) \delta: 2.39(\mathrm{~s}, 3 \mathrm{H}), 7.35(\mathrm{t}, J=8.0 \mathrm{~Hz}, 1 \mathrm{H}), 7.36(\mathrm{~d}$, $J=8.0 \mathrm{~Hz}, 1 \mathrm{H}), 7.41(\mathrm{~d}, J=8.0 \mathrm{~Hz}, 1 \mathrm{H}), 7.44(\mathrm{~s}, 1 \mathrm{H}) ;{ }^{13} \mathrm{C}$ NMR (125 MHz, $\left.\mathrm{CDCl}_{3}\right) \delta: 21.01,112.16,118.89,128.90$, 129.15, 132.35, 133.55, 139.14; GC-MS (EI, $70 \mathrm{eV}) \mathrm{m} / z$ : $117.01[\mathrm{M}]^{+}$.

2-甲基苯腈(2d ${ }^{[12 b]}$ : 无色液体. ${ }^{1} \mathrm{H}$ NMR $(500 \mathrm{MHz}$, $\left.\mathrm{CDCl}_{3}\right) \delta: 2.54(\mathrm{~s}, 3 \mathrm{H}), 7.27(\mathrm{t}, J=8.0 \mathrm{~Hz}, 1 \mathrm{H}), 7.32(\mathrm{~d}$, $J=8.0 \mathrm{~Hz}, 2 \mathrm{H}), 7.48$ (t, $J=7.5 \mathrm{~Hz}, 1 \mathrm{H}), 7.59$ (d, $J=7.5$ $\mathrm{Hz}, 2 \mathrm{H}) ;{ }^{13} \mathrm{C}$ NMR $\left(125 \mathrm{MHz}, \mathrm{CDCl}_{3}\right) \delta: 20.40,112.76$, $126.18,130.20,132.46,132.59,141.59$; GC-MS (EI, 70 eV) $m / z: 117.13[\mathrm{M}]^{+}$.

苯腈(2e) ${ }^{[1]}$ : 无色液体. ${ }^{1} \mathrm{H}$ NMR $\left(500 \mathrm{MHz}, \mathrm{CDCl}_{3}\right)$ $\delta: 7.46 \sim 7.49(\mathrm{~m}, 2 \mathrm{H}), 7.59 \sim 7.63(\mathrm{~m}, 1 \mathrm{H}), 7.64 \sim 7.66$ $(\mathrm{m}, 2 \mathrm{H}) ;{ }^{13} \mathrm{C}$ NMR $\left(125 \mathrm{MHz}, \mathrm{CDCl}_{3}\right) \delta: 112.39,118.76$, 129.06, 132.07, 132.71; GC-MS (EI, $70 \mathrm{eV}$ ) m/z: 103.11 $[\mathrm{M}]^{+}$.

4-叔丁基苯腈 $(2 \mathrm{f})^{[1]}$ : 浅黄色液体. ${ }^{1} \mathrm{H}$ NMR (500 $\left.\mathrm{MHz}, \mathrm{CDCl}_{3}\right) \delta: 1.33(\mathrm{~s}, 9 \mathrm{H}), 7.47 \sim 7.50(\mathrm{~m}, 2 \mathrm{H}), 7.57 \sim$ $7.59(\mathrm{~m}, 2 \mathrm{H}) ;{ }^{13} \mathrm{C}$ NMR $\left(125 \mathrm{MHz}, \mathrm{CDCl}_{3}\right) \delta: 30.86$, $35.18,109.27,119.04,126.10,131.88,156.58$; GC-MS
(EI, $70 \mathrm{eV}$ ) $\mathrm{m} / \mathrm{z}: 159.18[\mathrm{M}]^{+}$.

4-氟苯腈 $(2 \mathrm{~g})^{[12 b]}$ : 浅黄色固体, m.p. $29 \sim 30{ }^{\circ} \mathrm{C} ;{ }^{1} \mathrm{H}$ NMR $\left(500 \mathrm{MHz}, \mathrm{CDCl}_{3}\right) \delta: 7.16 \sim 7.21(\mathrm{~m}, 2 \mathrm{H}), 7.67 \sim$ $7.71(\mathrm{~m}, 2 \mathrm{H}) ;{ }^{13} \mathrm{C}$ NMR $\left(125 \mathrm{MHz}, \mathrm{CDCl}_{3}\right) \delta: 108.57$, 116.72, 117.96, 134.61, 163.89; GC-MS (EI, $70 \mathrm{eV}) \mathrm{m} / z$ : $121.02[\mathrm{M}]^{+}$.

4-氯苯腈 $(\mathbf{2 h})^{[12 b]}$ : 白色固体, m.p. 90 91 ${ }^{\circ} \mathrm{C} ;{ }^{1} \mathrm{H}$ $\operatorname{NMR}\left(500 \mathrm{MHz}, \mathrm{CDCl}_{3}\right) \delta$ : $7.47(\mathrm{~m}, 2 \mathrm{H}), 7.60(\mathrm{~m}, 2 \mathrm{H})$; ${ }^{13} \mathrm{C}$ NMR $\left(125 \mathrm{MHz}, \mathrm{CDCl}_{3}\right) \delta: 110.80,117.92,129.68$, 133.36, 139.54; GC-MS (EI, $70 \mathrm{eV}) \mathrm{m} / z: 137.01[\mathrm{M}]^{+}$.

3-氯苯腈 $(2 \mathrm{i})^{[39]}$ : 白色固体, m.p. $38 \sim 42{ }^{\circ} \mathrm{C} ;{ }^{1} \mathrm{H}$ NMR $\left(500 \mathrm{MHz}, \mathrm{CDCl}_{3}\right) \delta: 7.44(\mathrm{t}, J=8.0 \mathrm{~Hz}, 1 \mathrm{H})$, $7.55 \sim 7.57(\mathrm{~m}, 1 \mathrm{H}), 7.58 \sim 7.61(\mathrm{~m}, 1 \mathrm{H}), 7.64(\mathrm{~s}, 1 \mathrm{H}),{ }^{13} \mathrm{C}$ NMR $\left(125 \mathrm{MHz}, \mathrm{CDCl}_{3}\right) \delta: 114.00,117.38,130.27$, 130.47, 131.91, 133.20, 135.25; GC-MS (EI, $70 \mathrm{eV}) \mathrm{m} / \mathrm{z}$ : $136.96[\mathrm{M}]^{+}$.

2 -氯苯腈 $(\mathbf{2 j})^{[12 b]}$ : 浅黄色固体, m.p. $43 \sim 44{ }^{\circ} \mathrm{C} ;{ }^{1} \mathrm{H}$ NMR $\left(500 \mathrm{MHz}, \mathrm{CDCl}_{3}\right) \delta: 7.38$ (ddd, $J=8.25,7.25,1.5$ $\mathrm{Hz}, 1 \mathrm{H}), 7.51 \sim 7.57(\mathrm{~m}, 2 \mathrm{H}), 7.68(\mathrm{dd}, J=7.5,1.5 \mathrm{~Hz}$, $1 \mathrm{H}) ;{ }^{13} \mathrm{C}$ NMR $\left(125 \mathrm{MHz}, \mathrm{CDCl}_{3}\right) \delta: 113.52,115.93$, 127.14, 130.08, 133.83, 134.04, 136.95; GC-MS (EI, 70 eV) $m / z: 136.93[\mathrm{M}]^{+}$.

4-溴苯腈 $(\mathbf{2 k}){ }^{[2]}$ : 白色固体, m.p. $111 \sim 112{ }^{\circ} \mathrm{C} ;{ }^{1} \mathrm{H}$ NMR $\left(500 \mathrm{MHz}, \mathrm{CDCl}_{3}\right) \delta: 7.53(\mathrm{~d}, J=8.5 \mathrm{~Hz}, 2 \mathrm{H}), 7.64$ $(\mathrm{d}, J=8.5 \mathrm{~Hz}, 2 \mathrm{H}) ;{ }^{13} \mathrm{C}$ NMR $\left(125 \mathrm{MHz}, \mathrm{CDCl}_{3}\right) \delta$ : 111.26, 118.03, 128.01, 132.64, 133.40; GC-MS (EI, 70 eV) $m / z: 181.03[\mathrm{M}]^{+}$.

4-三氟甲基苯腈 $(2 \mathrm{l})^{[12 b]}$ : 白色固体, m.p. $41 \sim 42{ }^{\circ} \mathrm{C}$; ${ }^{1} \mathrm{H}$ NMR $\left(500 \mathrm{MHz}, \mathrm{CDCl}_{3}\right) \delta: 7.76(\mathrm{~d}, J=8.5 \mathrm{~Hz}, 2 \mathrm{H})$, $7.81(\mathrm{~d}, J=8.5 \mathrm{~Hz}, 2 \mathrm{H}) ;{ }^{13} \mathrm{C}$ NMR $\left(125 \mathrm{MHz}, \mathrm{CDCl}_{3}\right) \delta$ : $116.14,117.42,121.99,124.16,126.22,132.70,134.49$, 134.75; GC-MS (EI, $70 \mathrm{eV}$ ) m/z: $171.17[\mathrm{M}]^{+}$.

辅助材料(Supporting Information) 目标产物腈 2a 21 的气相色谱图、质谱、氢谱、碳谱. 这些材料可以免 费从本刊网站(http://sioc-journal.cn/)上下载.

\section{References}

[1] Tsuchiya, D.; Kawagoe, Y.; Moriyama, K.; Togo, H. Org. Lett. 2013, 15, 4194.

[2] Yadav, D. K. T..; Bhanage, B. M. Eur. J. Org. Chem. 2013, 23, 5106.

[3] Kawagoe Y.; Moriyama, K.; Togo, H. Eur. J. Org. Chem. 2014, 19, 4115.

[4] Wang, M.-X. Acc. Chem. Res. 2015, 48, 602.

[5] Nobuhito, K.; Takeshi, O. ACS Catal. 2016, 6, 989.

[6] Hodgson, H. H. Chem. Rev. 1947, 40, 251.

[7] Koelsch, C. F.; Whitney, A. G. J. Org. Chem. 1941, 06, 795.

[8] Takagi, K.; Okamoto, T.; Sakakibara, Y. Chem. Lett. 1973, 2, 471. 
[9] Ellis, G. P.; Romney-Alexander, T. M. Chem. Rev. 1987, 87, 779.

[10] Zanon, J.; Klapars, A.; Buchwald, S. L. J. Am. Chem. Soc. 2003, 125, 2890.

[11] Arvela, R. K.; Leadbeater, N. E. J. Org. Chem. 2003, 68, 9122.

[12] (a) Zhou, W.; Xu, J.-J.; Zhang, L.-R.; Jiao, N. Org. Lett. 2010, 12, 2888.

(b) Yu, Z.-W.; Li, L.-Y.; Shen, Z.-M. Chin. J. Org. Chem. 2017, 37, 1273.

[13] Rogic, M. M.; Van Peppen, J. F.; Klein, K. P.; Demmin, T. R. J. Org. Chem. 1974, 39, 3425.

[14] Yamaguchi, K.; Fujiwara, H.; Ogasawara, Y.; Kotani, M.; Mizuno, N. Angew. Chem. Int. Ed. 2007, 46, 3922.

[15] Rokade, B. V.; Prabhu, K. R. J. Org. Chem. 2012, 77, 5364.

[16] Denton, W. I.; Bishop, R. B.; Caldwell, H. P.; Chapman, H. D. Ind. Eng. Chem. 1950, 42, 796.

[17] Cavani, F.; Parrinello, F.; Trifiro, F. J. Molecuhr. Catal. 1987, 43, 117.

[18] Kumar, C. P.; Reddy, K. R.; Rao, V. V.; Chary, K. V. R. Green Chem. 2002, 4, 513.

[19] Zhang, Y.; Wang, J.-B. Angew. Chem. Int. Ed. 2013, 52, 10573.

[20] Murahashi, S.; Komiya, N.; Terai, H. J. Am. Chem. Soc. 2003, 125, 15312.

[21] Murahashi, S. I.; Nakae, T.; Terai, H.; Komiya, N. J. Am. Chem. Soc. 2008, 130, 11005.

[22] Liu, W-P.; Ackermann, L. Chem. Commun. 2014, 50, 1878.

[23] Do, H. Q.; Daugulis, O. Org. Lett. 2010, 12, 2517.

[24] Shu, Z.; Ji, W.; Wang, J. Angew. Chem. Int. Ed. 2014, 53, 2186.

[25] Li, J.; Ackermann, L. Angew. Chem. Int. Ed. 2015, 54, 3635.

[26] Gong, T.-J.; Xiao, B.; Fu, Y. J. Am. Chem. Soc. 2013, 135, 10630.

[27] Jiao, N. Nitrogenation Strategy for the Synthesis of N-containing Compounds, Springer, Singapore, 2017, p. 70.

[28] Chaitanya, M.; Yadagiri, D.; Anbarasan, P. Org. Lett. 2013, 15, 4960.

[29] Iida, S.; Togo, H. Tetrahedron 2007, 63, 8274.

[30] Rokade, B. V.; Malekar, S. K.; Prabhu, K. R. Chem. Commun.
2012, 48, 5506.

[31] Zhu, C.; Sun, C.; Wei, Y. Synthesis 2010, 4235.

[32] Vatèle J. M. Synlett 2014, 25, 1275.

[33] Ishida, T.; Watanabe, H.; Takei, T.; Hamasaki, A.; Tokunaga, M.; Harut, M. Appl. Catal. A. 2012, 425, 85.

[34] Oishi, T.; Yamaguchi, K.; Mizuno, N. Angew. Chem. Int. Ed. 2009, 48, 6286.

[35] Preger, Y.; Root, T. W.; Stahl, S. S. ACS Omega 2018, 3, 6091

[36] Yadav, D. K. T.; Bhanage, B. M. Eur. J. Org. Chem. 2013, 23, 5106.

[37] Wu, Y.-X.; Xi, Y.-C.; Zhao, M.; Wang, S.-Y. Chin. J. Org. Chem. 2018, 38, 2590.

[38] Qu, Q.-H.; Gao, X.-F.; Gao, J.; Yuan, G.-Q. Sci. Chin. Chem. 2015, 58,747 .

[39] Fan, Z.-Q.; Yang, X.-J.; Shen, Z.-L.; Li, M.-C. J. Electrochem. Soc. 2017, 164, 54.

[40] Zhu, Y.-H.; Zhu, Y.; Zeng, H.-Y.; Chen, Z.-Y.; R. D. Little.; Ma, C.-A. J. Electroanal. Chem. 2015, 751, 105.

[41] Zhu, Y.-H.; Chen, Z.-Y.; Zhang, J.-Q.; Wu, Q.-Q.; Ma, C.-A.; Little, R. D. Electrochim. Acta 2016, 207, 308.

[42] Chiou, S.; Hoque, A. K. M. M.; Shine H. J. J. Org. Chem. 1990, 55, 3227.

[43] Zhang, L.; Chen, H.; Zha, Z.-G.; Wang, Z.-Y. Chem. Commun. 2012, 48, 6574.

[44] Meng, L.; Su, J.-H.; Zha, Z.-G.; Zhang, L.; Zhang, Z.-L.; Wang, Z.-Y. Chem. Eur. J. 2013, 19, 5542.

[45] Zhao, H.-Bo.; Xu, P.; Song, J.-S.; Xu, H.-C. Angew. Chem. Int. Ed. 2018, 57, 15153.

[46] Petrosyan, V. A.; Niyazymbetov, M. E.; Ul'yanova, E. V. Russ. Chem. Bull. 1989, 38, 1548.

[47] Petrosyan, V. A.; Niyazymbetov, M. E.; Ul'yanova, E. V. Russ. Chem. Bull. 1990, 39, 546.

[48] Hartmera, M. F.; Waldvogel, S. R. Chem. Commun. 2015, 51, 16346

(Li, L.; Fan, Y.) 